\section{ACHIEVING HIGH RELIABILITY THROUGH CARE COORDINATION FOR PATIENTS WHO REQUIRE EMERGENCY SURGERY}

Sapan Desai, John Cosentino, Kimberly Nagy. Northwest Community Hospital, US

\subsection{6/bmjoq-2017-IHI.1}

Background An abdominal aortic aneurysm is a balloon-like swelling in the largest blood vessel of the body. AAAs are present in $10 \%$ of the population, and a ruptured AAA is associated with a $90 \%$ risk of death and $\$ 150000$ cost of care.

Objectives The goal of our project is to streamline the process for getting a patient with a ruptured aneurysm to the operating room (OR) from the time they present in the emergency department (ED).

Methods Our implementation strategy included the use of lean six sigma tools and methodologies, intentional clinical process design, team-based training, computer modelling, and live simulation.

Results The impact of this work has been a decrease in time from $\mathrm{ED}$ to OR (217 to $45 \mathrm{~min}$ ), leading to a $224 \%$ decline in complications, and decrease in LOS (15.2 to 5.4 days). Survival has dramatically increased to $90 \%$. These changes have led to a $\$ 1.8$ million dollar impact on operations, and our performance has improved from the $70 \%$ percentile to the top $1 \%$ in the country over the past year.

Conclusions The main contributing factors for this improved care coordination centres around streamlining the process map from 172 steps to 33 steps, simplifying the communication matrix, cross training of staff to better understand each person's role in patient care, and applying a control plan with close follow. The impact has been to successfully achieve the six aims of the Institute of Medicine, deliver world class care to our community, and espouse the mission and values of our healthcare system.

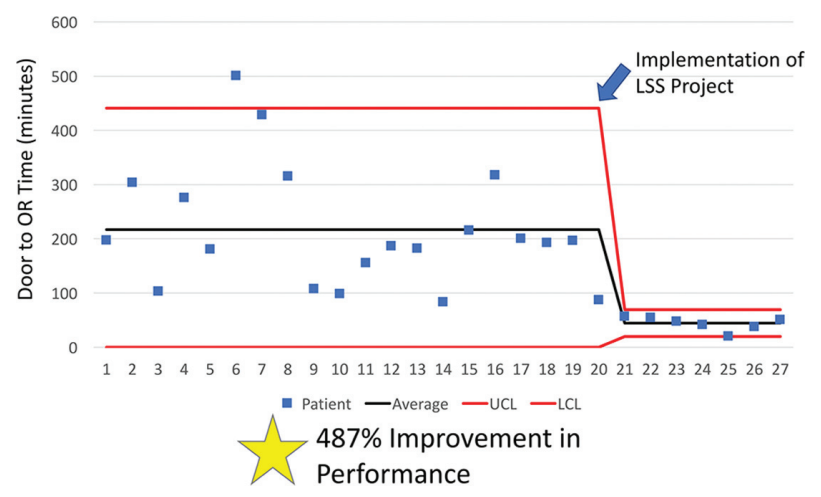

Abstract 837 Figure 1 Door to OR control chart $(4 / 2015-6 / 2017)$



Abstract 837 Figure 2 Length of stay $(4 / 2015-6 / 2017)$



Abstract 837 Figure 3 rAAA patient flow process - future state 